\title{
ИСПОЛЬЗОВАНИЕ СИСТЕМ С ИСКУССТВЕННЫМ ИНТЕЛЛЕКТОМ В ДИАГНОСТИКЕ ИНФЕКЦИЙ, ПЕРЕДАЮЩИХСЯ ПОЛОВЫМ ПУТЕМ
}

\section{THE USE OF ARTIFICIAL INTELLIGENCE SYSTEMS IN THE DIAGNOSIS OF SEXUALLY TRANSMITTED INFECTIONS}

A. Smetankina

Summary. The modern direction of clinical diagnostics is to assess the state of the body, individual organs and systems by the presence of certain gases and vapors of substances associated with the disease in the bioassays.

Keywords: bioprobes, artificial systems, piezo-quartz microweaving, artificial intelligence, multisensory systems.

\author{
Сметанкина Анастасия Васильевна \\ Аспирант, Воронежский государственный \\ медицинский университет им. Н. Н. Бурденко \\ Zhuchkova954@yandex.ru
}

Аннотация. Современным направлением клинической диагностики является оценка состояния организма, отдельных органов и систем по наличию определенных газов и паров веществ, связанных с заболеванием в биопробax.

Ключевые слова: биопробы, искусственные системы, пьезокварцевое микровзвешивание, искусственный интеллект, мультисенсорные системы.

С целью улучшения диагностики предложен метод экспресс-диагностики наличия определенного вида инфекций, передающихся половым путем, заключающийся в качественном определении легколетучих органических соединений в биопробах.

\section{Результаты и обсужление}

Аналитический сигнал мультисенсорной системы представляет собой совокупность выходных кривых массива сенсоров (хроночастотограммы) - зависимость изменения частоты колебаний пьезокварцевой пластины с модификатором от времени или хроночастотограммы. При этом не все точки на выходных кривых информативны и используются для получения аналитической информации. Визуализация выходного сигнала осуществляется путем построения «визуальных отпечатков» в двух вариантах: по максимальным сигналам всех сенсоров в матрице («визуальные отпечатки» максимумов) и по отдельным сигналам всех сенсоров, регистрируемых по определенному алгоритму (кинетические «визуальные отпечатки»), что предусмотрено в программном обеспечении анализатора «МАГ-8». Геометрические особенности «визуальных отпечатков» (форма, площадь фигуры) определяются качественным и количественным (соотношение содержаний и концентрация отдельных соединений) составом РГФ над анализируемыми биопробами. Рассмотрим наиболее типичные «визуальные отпечатки» для различных групп биопроб пациенток с разной диагностической характеристикой по природе инфекции или ее отсутствии: контрольная группа - «клинически здоровые», «пролеченные», об- 
Кинетические

«визуальные отпечатки»

$\Delta \mathrm{F}, \Gamma \mathrm{L}$

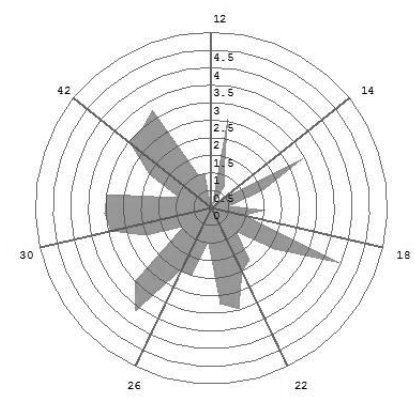

a)
«Визуальные отпечатки»

максимумов

$\Delta \mathrm{F}, \Gamma ц$

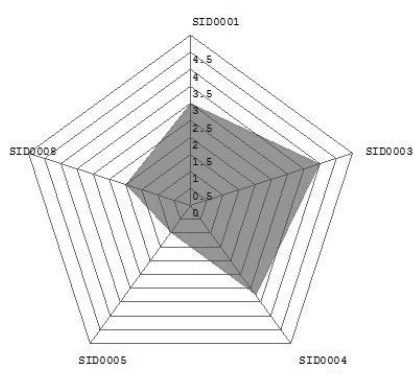

6)

«Клинически здоровые», $S_{6 . o .}=557$ Гц·с

$\Delta \mathrm{F}, Г ц$

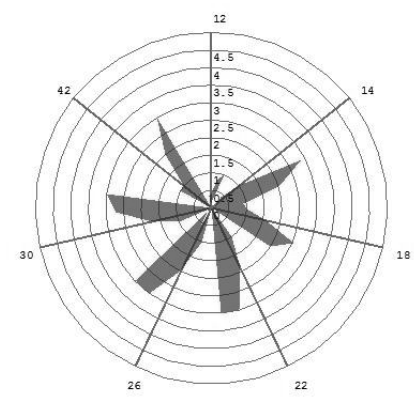

a)
$\Delta \mathrm{F}, \Gamma ц$

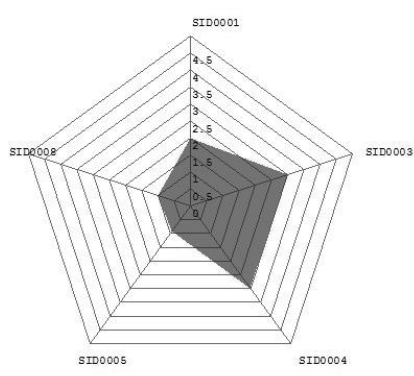

б)

«Пролеченные», $S_{\text {6.o. }}=399$ Гц·с

Рис. 1. Кинетические «визуальные отпечатки» (а) и «визуальные отпечатки» максимумов сигналов (б) массива 5-ти сенсоров в РГФ над биопробами контрольной группы.

следуемая группа — «кандидоз», «гарднереллез», «уреаплазмоз», «хламидиоз», «вирус папилломы человека» (рис. 1, 2). Для детализации тонких различий в форме «визуальных отпечатков» отключали сенсоры с маленькими (на уровне шума) и наибольшими сигналами, при этом число сенсоров, по откликам которых строили «визуальные отпечатки» составило 5: ПЭГ-2000, ДЦГ-18-К-6, ТХ-100, БКЗ, ПЭГад.

Для проб контрольной группы характерны различия в геометрии кинетических «визуальных отпечатков»: так для проб из подгруппы «пролеченные» характерны меньшие сигналы массива сенсоров в РГФ, чем для подгруппы «клинически здоровые», что можно объяснить угнетением естественной микрофлоры вследствие проведенного лечения и, как следствие, уменьшением содержания легколетучих органических веществ маркеров в РГФ над биопробой. Также для подгруппы «клинически здоровые» характерны более четкие «лепестки» на кинетическом «визуальном отпечатке» в начальные моменты времени сорбции (10 с от момента инжектирования РГФ в ячейку детектирования), а для подгруппы «пролеченные» - после 30 с от начала сорбции.

\section{Зак^ючение}

«Визуальные отпечатки» максимумов откликов сенсоров в РГФ над биопробами для контрольной группы практически не отличаются по геометрии, что 
Кинетические

«визуальные отпечатки»

$\Delta \mathrm{F}, \Gamma \mathrm{L}$

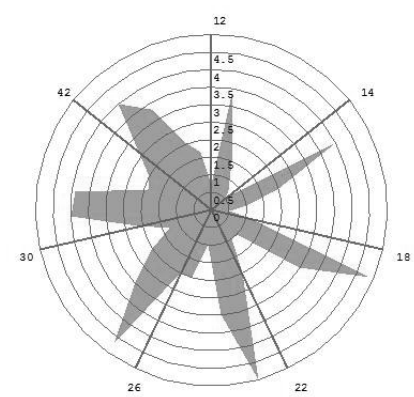

a)

«Кандидоз», $S_{\text {6.o. }}=711$ Гц·с

$\Delta \mathrm{F}, \Gamma \mathrm{L}$

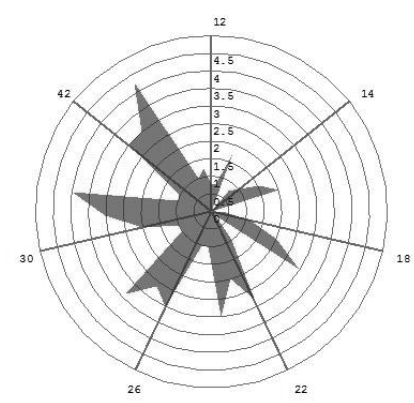

a)

«Гарднереллез», $S_{\text {6.o. }}=792$ Гц·с

$\Delta \mathrm{F}, \Gamma \mathrm{L}$

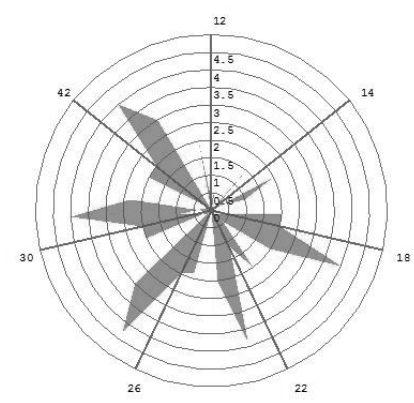

a)

«Визуальные отпечатки» максимумов

$\Delta \mathrm{F}, \Gamma ц$

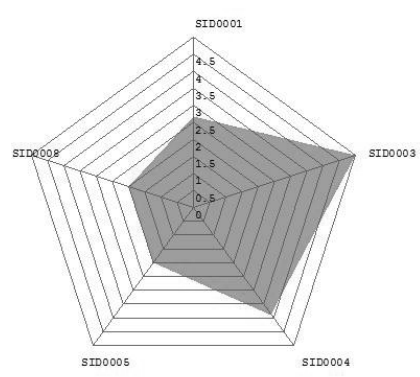

б)

$\Delta \mathrm{F}$, Гц

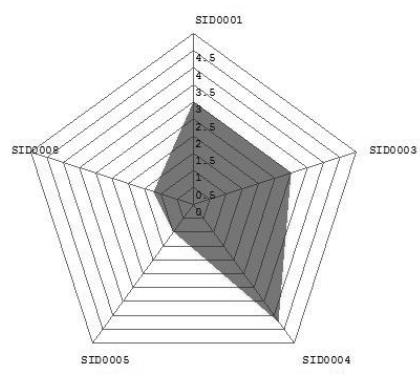

б)

$\Delta \mathrm{F}$, Гц

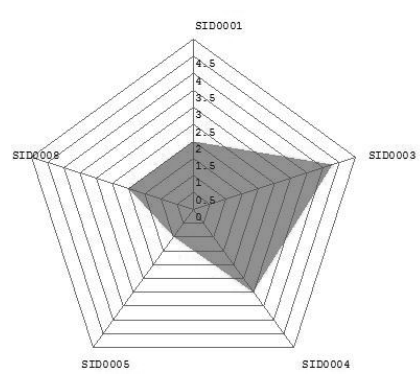

б)

«Гарднереллез», $S_{\text {6.o. }}=792$ Гц·с

Рис. 2. Типичные кинетические «визуальные отпечатки» (а) и максимумов (б) откликов массива сенсоров в РГФ над цервикальной слизью для обследуемой группы пациенток с моноинфекциями (продолжение на стр. 84 
$\Delta \mathrm{F}, \Gamma \mathrm{L}$

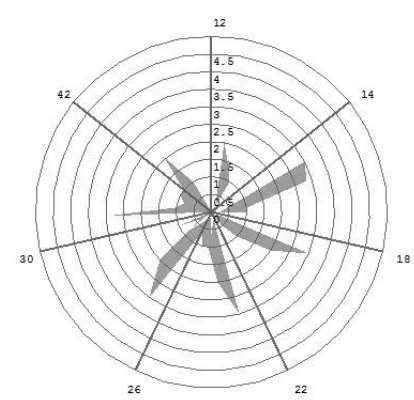

a)

$\Delta \mathrm{F}, \Gamma \mathrm{L}$

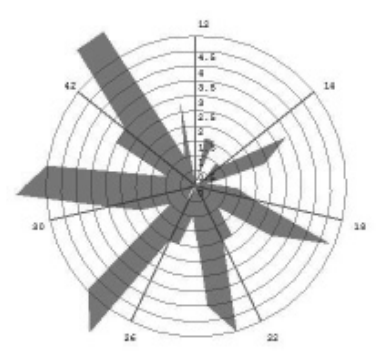

a)
$\Delta \mathrm{F}$, Гц

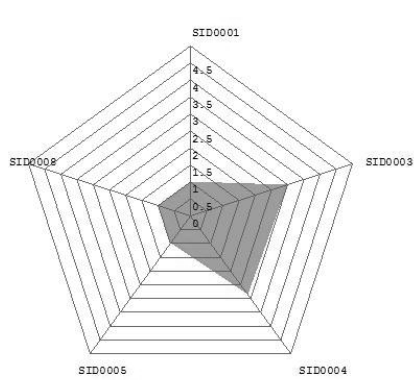

б)

«Хламидиоз», $S_{\text {6.o. }}=371$ Гц·с

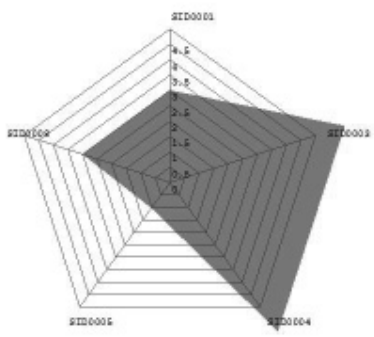

б)

«Вирус папилломы человека» («ВПЧ»), $S_{6 . o .}=1090$ Гц·с

$\Delta \mathrm{F},\ulcorner ц$

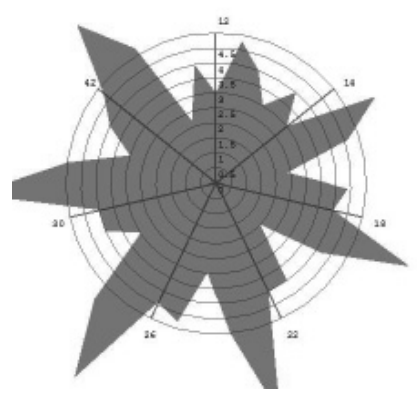

a)

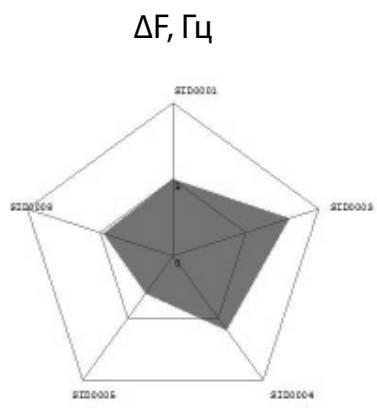

б)

«Микстинфекция» (кандидоз, гарднереллез, хламидиоз, уреаплазмоз)

$$
S_{\text {6.o. }}=1317 \text { Гц·с }
$$

Рис. 2 (продолжение). Типичные кинетические «визуальные отпечатки» (а) и максимумов (б) откликов массива сенсоров в РГФ над цервикальной слизью для обследуемой группы пациенток

с моноинфекциями

свидетельствует об идентичности состава РГФ над биопробами и низком диагностическом свойстве этого вида сигналов. Также для образцов контрольной группы характерны меньшие по значению отклики выбранных сенсоров в РГФ над ними, чем для основной группы, и ге- ометрия «визуального отпечатка» максимумов без четко выраженного смещения в сторону откликов одного сенсора. Однако для «визуального отпечатка» откликов массива сенсоров в РГФ над цервикальной слизью с заболеванием «хламидиоз» характерна самая маленькая 
площадь из рассматриваемых, что может быть связано с отсутствием возбудителя в отбираемом материале для анализа и, как следствие, отсутствием газов-маркеров В РГФ над ним. Этим объясняется невозможность диагностики возбудителя из этой группы с помощью простых алгоритмов (уровень 1 и 2).

Для основной группы характерны различные «визуальные отпечатки» откликов массива сенсоров в РГФ над биопробами не только соответствующие различным заболеваниям, но и внутри группы для одного заболевания. Это может быть связано с повышенным влиянием матрицы биопробы на состав РГФ, т.к. одно и то же заболевание может вызывать различные нарушения в микрофлоре цервикального канала, вследствие чего различия между «визуальными отпечатками» над био- пробами для ИППП нивелируются, что затрудняет определение вида возбудителя.

Для всех исследуемых возбудителей ИППП за исключением кандид и хламидий характерен «разворачивающийся» вид кинетического «визуального отпечатка», т.е. увеличение сигналов массива сенсоров со временем, что свидетельствует о большом содержании газов-маркеров в РГФ над биопробой и активном протекании заболевания. Кинетический «визуальный отпечаток» откликов массива сенсоров в РГФ над биоматериалом содержащим грибки рода кандида похож на кинетический «визуальный отпечаток» для проб из группы «клинически здоровые» и не может быть выделен в отдельную группу. Поэтому даже по визуализации второго уровня пробы с этим возбудителем не диагностируются.

\section{ЛИТЕРАТУРА}

1. Revelsky A.I., Revelsky I. A., Miller B., Oriedo V. Simultaneous determination of fatty, dicarboxylic and amino acids based on derivatization with isobutyl chloroformate followed by gas chromatography — positive ion chemical ionization mass spectrometry //J. Chromatogr. B. 2004. V. 800. № 1-2. P. 101.

2. Сухарев А., Ермолаева Т. Н., Беда Н. А., Мамаева С. А., Ермолаев В. Г. Иммунохимическое исследование лактоферрина, продуктов деградации фибриногена, иммуноглобулинов и белковых фракций в смешанной слюне перспективный неинвазивный тест в оценке состояния здоровья при диспансеризации // Современные наукоемкие технологии. 2006. № 6. С. 79.

3. Pavlou A.K., Magan N., McNulty C., Jones J. M., Sharp D., Brown J., Turner A. P.F. Use of an electronic nose system for diagnoses of urinary tract infections// Biosens. Bioelectron. 2002. V. 17. № 10. P. 893.

4. Bernabei M., Pennazza G., Santonico M., Corsi C., Roscioni C., Paolesse R., Di Natale C., D'Amico A. A preliminary study on the possibility to diagnose urinary tract cancers by an electronic nose // Sens. Actuators B: Chem. 2008. V. 131. № 1. P. 1.

5. Бельский В. Е. Солюбилизация газов и насыщенных паров в биологических средах // Хим.-фарм. журнал. 2000. Т. 34. № 11. С. 42.

6. Buszewski B., Ulanowska A., Kowalkowski T., CieslinskiK. Identification of volatile lung cancer markers by gas chromatography-mass spectrometry: Comparison with discrimination by canines // Anal. Bioanal. Chem. 2012. V. 404. № 1-2. P. 141.

7. Gaspar E.M., Lucena A.F., Duro da Costa J., Chavesdas Neves H. Organic metabolites in exhaled human breath — A multivariate approach for identification of biomarkers in lung disorders // J. Chromatogr. A. 2009. V. 1216. № 14. P. 2749.

8. Gardner J.W., Shin H. W., Hines E. L. An electronic nose system to diagnose illness // Sens. Actuators B: Chem. 2000. V. 70. № 1-3. P. 19.

9. Кучменко Т.А., Шуба А. А., Тюркин И.А, Битюкова В. В. Оценка состояния биологических проб по составу равновесной газовой фазы спримене нием мультисенсорной системы // Журн. аналит. химии. 2014. Т. 69. № 5. С. 534. (Kuchmenko Т. A., Shuba A. A., Tyurkin I. A., Bityukova V. V. Estimation of the state of biological samples by the composition of the headspace using a multisensor system. // J. Analyt. Chem. 2014. V. 69. № 5. P. 485.)

10. Weiss T., Angerer J. Simultaneous determination of various aromatic amines and metabolites of aromatic nitro compounds in urine for low level exposure using gas chromatography-mass spectrometry // J. Chromatogr. B. 2002. V. 778. № 1-2.P. 179.

11. Deng Y.-H., Wang H., Zhong L., Zhang H.-S. Trace determination of short-chain aliphatic amines in biological samples by micellar electrokinetic capillary chromatography with laser-induced fluorescence detection // Talanta. 2009. V. 77. № 4. P. 1337.

12. Rudnicka J., Kowalkowski T., Ligor T., Buszewski B. Determination of volatile organic compounds as biomarkers of lung cancer by SPME-GC-TOF/MS and chemometrics // J. Chromatogr. B. 2011. V. 879. № 30. P. 3360.

13. Senthilmohan S.T., Kettle A. J., McEwan M.J, Dummer J., Edwards S. J., Wilson P. F., Epton M. J. Detection of monobromamine, monochloramine and dichloramine using selected ion flow tube mass spectrometry and their relevance as breath markers // Rapid Commun. Mass Spectrom. 2008. V. 22. № 5. P. 677.

14. Carrola J, Rocha C. M., Barros A. S., Gil A. M., Goodfellow B. J., Carreira I. M., Bernardo J., Gomes A., Sousa V., Carvalho L., Duarte I. F. Metabolic signatures of lung cancer in biofluids: NMR-based metabonomics of urine // J. Proteome Res. 2011. V. 10. № 1. P. 221. 